\title{
Relationship between anthropometrics and handgrip strength among Nigerian school children
}

\author{
Adebisi I. Hammed, Elvis I. Agbonlahor \\ Department of Human Kinetics and Sports Science, University of Benin, Benin City
}

\section{Summary}

Study aim: Was to investigate the relationship of anthropometrics with handgrip strength (HGS) among Nigerian school children.

Material and methods: A total of 200 school children participated in this study. An electronic handgrip dynamometer was used to measure handgrip strength (HGS) in $\mathrm{kg}$. Body height and body weight were measured with a wall-mounted stadiometer in meters and a bathroom weighing scale in $\mathrm{kg}$, respectively. Body mass index (BMI) was then calculated using the formula weight $(\mathrm{kg}) /$ height $\left(\mathrm{m}^{2}\right)$. Also, hand span of both hands was measured from the tip of the thumb to the tip of the small finger with the hand opened as wide as possible. The distance from the distal wrist crease up to the base of the middle finger was taken for palm length and the distal wrist crease to the tip of the middle finger was considered for the measurement of hand length. However, the relationship between HGS and anthropometric parameters was analyzed using Pearson's product moment coefficient of correlation.

Results: The outcome of this study showed that hand and palm length correlated disproportionately and insignificantly ( $\mathrm{p}>0.05)$ with both dominant and non-dominant HGS. Also, hand span was found to be a disproportionate correlate of HGS, though such a correlation was only significant $(\mathrm{p}<0.05)$ with dominant HGS. However, BMI and handedness were observed to associate proportionately and significantly $(\mathrm{p}<0.05)$ with HGS.

Conclusion: This study therefore concluded that the most important determinants of HGS among Nigerian school children are BMI, handedness and hand span, and thus could be considered as markers of nutritional and health status, as well as physical fitness of these individuals.

\section{Keywords: Anthropometric properties - Hand strength and grade-schoolers}

\section{Introduction}

Reliable and valid evaluation of hand strength can provide an objective index of general upper body strength. Grip strength is the result of forceful flexion of the finger joints with a maximal voluntary force that a person is able to exert under normal biokinetic conditions. Hand grip strength (HGS) is an important component to perform precise and refined fine motor activities. These fine motor skills start developing as a child grows, but precise need of these skills increases when the child starts performing activities such as drawing, feeding and so on. As the child grows, there will be increased demand for performing fine motor activities, which forms an integral part of the child's motor development. HGS is one of the commonest measurements used as a part of physical assessment of various diseases affecting musculoskeletal, neuromuscular and cardiorespiratory systems in children, elderly and obese populations. It also provides selective information to estimate the nutritional status and overall muscle strength of an individual [13].

Meanwhile, a decrease in HGS can cause significant functional limitations which could lead to decreased basic and instrumental activities of daily living, thereby diminishing quality of life of an individual. This is because hand functionality is considered to be vital in most of the activities involving the upper limbs, such as writing, eating, carrying loads, lifting objects, opening or closing doors, to name a few. Most sports activities also require adequate grip strength to enhance performance and prevent overuse injuries that are closely associated with inadequate grip strength [12]. Many factors influence the strength of the grip, including muscle strength, fatigue, time of the day, 
age, gender, nutritional status, restricted motion, anthropometric parameters and pain [10]. Grip strength is often used in medicine as a specific test for hand strength. The purpose of this testing is diverse, including to diagnose diseases, to evaluate and compare treatments, to document progression of muscle strength, and to provide feedback during the rehabilitation process as a measure indicating the level of hand function and fatigue.

Several studies have highlighted the influence of age, gender, body size, body height and body weight on HGS among children, particularly in the early stage of puberty $[8,9,11]$. Body weight and stature (body height) are primary indicators of human growth, and also highly significant relationships between maximal HGS of the dominant hand and general anthropometric variables in all age groups have been reported [17]. A parallel increase of HGS has been demonstrated with age, which seems largely dependent on the increase in body mass index (BMI) and, particularly, muscle mass. However, this could simply reflect the sexual dimorphism, due to the action of sex steroid hormones. Furthermore, evidence has shown that there were strong correlations between grip strength and various anthropometric traits, such as weight, height, hand length and BMI, as had been reported earlier [14, 15]. In fact, the grip strength has been reported to be higher in the dominant hand among children and adult populations [7]. Right and left HGS were positively correlated with weight, height and body surface area among the Caucasian population [6]. In effect, many HGS studies in young healthy individuals have revealed that anthropometrics such as body height, body weight, BMI, hand length, hand circumference and grip span are positively associated with HGS [16]. A significant positive correlation between BMI and HGS and handgrip endurance in different weight groups among apparently healthy male and female individuals has been reported [3]. Similarly, a significant positive correlation of dominant HGS with age, body height, body weight, BMI and hand span of the dominant hand in males and females has also been found [1]. It has also been observed that persons with longer fingers and larger hand surface areas have stronger grip power. In addition, hand length, palm width or length, forearm length, forearm circumference and wrist circumference were significantly associated with HGS differently among populations [4].

The relation of the anthropometric variables with HGS among Nigerian school children has not been properly explored to determine the influence of the former on the latter. Also, there are a few studies in Nigeria that have attempted to establish the relationship between HGS and anthropometric data, particularly the hand span, hand length and palm length, among others, in this population. This study therefore aims to evaluate the relationship of anthropometric data with HGS among Nigerian school children.

\section{Research hypotheses}

The following hypotheses were formulated and tested at the 0.05 alpha level:

1. There will be no significant relationship between BMI and HGS among Nigerian school children.

2. Hand dominance (handedness) will not significantly correlate with HGS among Nigerian school children.

3. There will be no significant relationships between hand length and HGS, or palm length and HGS, among Nigerian school children.

4. There will be no significant association between hand span and HGS among Nigerian school children.

5. Gender will not significantly influence HGS among Nigerian school children.

\section{Material and methods}

\section{Research design}

This study was a correlational survey of the interface of anthropometrics and HGS among Nigerian school children.

\section{Population}

The population for this study included Oyo state school children between the biological ages of 7 to 10 years from three primary schools in the 2015/2016 academic session.

\section{Sample size and sampling technique}

A total of one hundred (100) male and one hundred (100) female school children participated in this study. They were recruited using the proportionate random sampling technique. However, participants with any joint problems of the hand, wrist and elbow, history of fracture, neurological disorder, and deformities of the upper limb were excluded from the study.

\section{Measurements and data collection}

The study received ethical approval from the Research Ethics Committee of the University of Benin for permission to conduct this study and the subjects were then recruited consecutively. HGS was measured using a Camry Electronic Hand Dynamometer (Model: EH101). It comes with dual scale readout of forces in kilograms and pounds. However, all readings were recorded in kilograms in the present study. To standardize the test, the following guidelines were established by two evaluators: the arm positioning followed the American Society of Hand Therapists guidelines [5], with the subject comfortably seated with the shoulder slightly forward and the elbow flexed at a $90^{\circ}$ angle. The forearm and wrist were in a neutral position. Three maximum efforts were performed alternately for each arm, with three-second contractions and ten-second rest periods between the attempts. The best of 
three attempts was recorded by the evaluators. However, the following test instructions were provided: "you must squeeze the handle as hard as possible keeping both your body and arm in position". The same tone was used during the briefings, and no verbal encouragement was offered. The instrument calibration was assessed periodically throughout the study. Also, age and hand dominance (the one which is preferred for daily activities such as writing and eating) were recorded.

The standing heights were measured using a stadiometer. Each participant was assessed while in a good standing posture on the foot resting on the device with minimal clothing without shoes but with the head facing forward in the front font position. The subjects also had the shoulders relaxed, arms hanging loosely on both sides, palms facing forward, feet together and knees straight. The height for each participant was taken when the movable headboard was lowered to touch the crown of the head. The measurements were taken to the nearest $0.1 \mathrm{~cm}$. The calibrated weighing scale was used to measure body weight and was checked for zero balance before each use. Participants were instructed to empty their pockets and remove shoes and any apparel that could interfere with weight measurements. They then stood on the scale looking straight ahead, relaxed and remained motionless without leaning on any object or the wall. Weight measurements were taken when the scale stabilized and recorded to the nearest $0.1 \mathrm{~kg}$ while protecting the confidentiality of the participants' values. BMI was then calculated using the formula weight $(\mathrm{kg}) /$ height $\left(\mathrm{m}^{2}\right)$. Hand dimension measurements were made by a standard flexible measuring tape to the nearest centimeter with the hand extended and relaxed while the elbow was supported on a table. Hand span was measured in both hands from the tip of the thumb to the tip of the small finger with the hand opened as wide as possible. The perimeter of the middle part of the hand was considered for palm length. For palm length, the distance from the distal wrist crease up to the base of the middle finger, and lastly, for the hand length, the distal wrist crease to the tip of the middle finger was considered for the measurement.

\section{Data analysis}

The data obtained from this study were analyzed using descriptive and inferential statistics. The demographic and anthropometric profile of the participants was summarized using frequency counts, percentages, mean and standard deviation. The relationship between HGS and anthropometrics was analyzed using the Pearson product moment coefficient of correlation, and then differences in anthropometrics across gender categories were analyzed using the independent sample t-test. Statistical significance was accepted for a $p$ value of $<0.05$. All the analyses were performed using the Statistical Package for the Social Sciences (SPSS) version 20.0.

\section{Results}

The results are presented in Tables 1-3.

The results of this study showed that of all the anthropometric parameters evaluated, only hand and palm length were not significantly associated with HGS. Factors such as gender, BMI, handedness and hand span correlated significantly with HGS.

Table 1. Descriptive statistics for general samples $(N=200)$

\begin{tabular}{lcc}
\hline Variables & $\mathrm{n}$ & Mean $\pm \mathrm{SD}$ \\
\hline Gender & & - \\
Male & 100 & - \\
Female & 100 & $11.05 \pm 0.82$ \\
Age [years] & - & $1.35 \pm 0.63$ \\
BH [m] & - & $26.95 \pm 4.22$ \\
BW [kg] & - & $14.71 \pm 1.89$ \\
BMI [kg/m $\left.{ }^{2}\right]$ & - & $3.99 \pm 0.75$ \\
DHGS [kg] & - & $3.82 \pm 0.82$ \\
NDHGS [kg] & - & $16.46 \pm 1.20$ \\
DHS [cm] & - & $16.43 \pm 1.21$ \\
NDHS [cm] & - & $15.52 \pm 1.24$ \\
DHL [cm] & - & $15.46 \pm 1.24$ \\
NDHL [cm] & - & $9.03 \pm 0.65$ \\
DPL [cm] & - & $9.01 \pm 0.64$ \\
NDPL [cm] & - & \\
\hline
\end{tabular}

$\mathrm{n}$ - frequency within a group or subgroup, $\mathrm{BH}$ - body height, $\mathrm{BW}$ - body weight, BMI - body mass index, DHGS - dominant handgrip strength, NDHGS - non dominant handgrip strength, DHS - dominant hand span, NDHS - non dominant hand span, DHL - dominant hand length, NDHL - non dominant hand length, DPL - dominant palm length, NDPL - non dominant palm length.

\section{Hypothesis testing}

\section{Hypothesis 1}

There will be no significant relationship between BMI and HGS among Nigerian school children.

This study showed that BMI is a proportionate (DHGS, $\mathrm{r}=0.454$; NDHGS, $\mathrm{r}=0.445)$ and significant $(\mathrm{p}<0.05)$ correlate of HGS and therefore the null hypothesis $\left(\mathrm{H}_{0}\right)$ was rejected. This is an indication that BMI is a predictor of HGS among Nigerian primary school students.

\section{Hypothesis 2}

Hand dominance (handedness) will not significantly correlate with HGS among Nigerian school children.

It was observed from this study that a proportionate $(\mathrm{r}=0.850)$ and significant $(\mathrm{p}<0.05)$ difference exists 
Table 2. Descriptive statistics and independent samples t-test for male and female samples

\begin{tabular}{|c|c|c|c|c|}
\hline Variables & $\begin{array}{c}\text { Male }(n=100) \\
\text { Mean } \pm S D\end{array}$ & $\begin{array}{c}\text { Female }(n=100) \\
\text { Mean } \pm \text { SD }\end{array}$ & $\mathrm{t}$ & Sig (2-tailed) \\
\hline Age [years] & $11.03 \pm 1.17$ & $11.07 \pm 1.15$ & -0.244 & 0.807 \\
\hline $\mathrm{BH}[\mathrm{m}]$ & $1.35 \pm 0.60$ & $1.36 \pm 0.61$ & -0.460 & 0.646 \\
\hline $\mathrm{BW}[\mathrm{kg}]$ & $27.37 \pm 3.77$ & $26.53 \pm 4.60$ & 1.411 & 0.160 \\
\hline BMI $\left[\mathrm{kg} / \mathrm{m}^{2}\right]$ & $15.01 \pm 1.77$ & $14.42 \pm 1.98$ & 2.220 & $0.028^{*}$ \\
\hline DHGS [kg] & $4.11 \pm 0.86$ & $3.86 \pm 0.60$ & 2.444 & $0.015^{*}$ \\
\hline NDHGS [kg] & $3.96 \pm 0.88$ & $3.67 \pm 0.73$ & 2.564 & $0.011^{*}$ \\
\hline DHS [cm] & $16.44 \pm 1.21$ & $16.48 \pm 1.20$ & -0.287 & 0.774 \\
\hline NDHS [cm] & $16.41 \pm 1.22$ & $16.45 \pm 1.20$ & -0.275 & 0.784 \\
\hline $\mathrm{DHL}[\mathrm{cm}]$ & $15.32 \pm 1.10$ & $15.72 \pm 1.36$ & -2.303 & $0.022 *$ \\
\hline NDHL $[\mathrm{cm}]$ & $15.26 \pm 1.09$ & $15.67 \pm 1.34$ & -2.403 & $0.017 *$ \\
\hline $\mathrm{DPL}[\mathrm{cm}]$ & $9.02 \pm 0.60$ & $9.05 \pm 0.70$ & -0.304 & 0.762 \\
\hline NDPL [cm] & $9.00 \pm 0.60$ & $9.03 \pm 0.70$ & -0.308 & 0.759 \\
\hline
\end{tabular}

$\mathrm{n}$ - frequency within a group or subgroup, BH - body height, BW - body weight, BMI - body mass index, DHGS - dominant handgrip strength, NDHGS - non dominant handgrip strength, DHS - dominant hand span, NDHS - non dominant hand span, DHL - dominant hand length, NDHL - non dominant hand length, DPL - dominant palm length, NDPL - non dominant palm length, t-test statistics, Sig (2-tailed) - two-tailed p-value evaluating the null against an alternate that the mean is not equal to 50 .

Table 3. Correlations of Participants' Anthropometric Parameters with HGS

\begin{tabular}{|c|c|c|c|c|c|c|c|c|c|c|c|c|c|}
\hline & Age & Gender & $\mathrm{BH}$ & BW & BMI & DHGS & NDHGS & DHS & NDHS & DHL & NDHL & DPL & NDPL \\
\hline Age & 1 & & & & & & & & & & & & \\
\hline Gender & 0.017 & 1 & & & & & & & & & & & \\
\hline $\mathrm{BH}$ & 0.017 & 0.033 & 1 & & & & & & & & & & \\
\hline BW & -0.007 & -0.100 & $0.588^{*}$ & 1 & & & & & & & & & \\
\hline BMI & -0.073 & $-0.156^{*}$ & -0.018 & $0.794 * *$ & 1 & & & & & & & & \\
\hline DHGS & $-0.141^{*}$ & $-0.171^{*}$ & 0.019 & 0.350 ** & $0.454 * *$ & 1 & & & & & & & \\
\hline NDHGS & $-0.142 *$ & $-0.179^{*}$ & 0.017 & $0.360^{* *}$ & $0.445^{* *}$ & $0.850^{* *}$ & 1 & & & & & & \\
\hline DHS & $0,212^{* *}$ & 0,020 & 0,021 & $-0,043$ & -0.082 & $-0.177^{*}$ & -0.100 & 1 & & & & & \\
\hline NDHS & $0.218^{* *}$ & 0.020 & 0.023 & -0.040 & -0.079 & $-0.182 * *$ & -0.103 & $0.999 * *$ & 1 & & & & \\
\hline DHL & $0.284 * *$ & $0.162 *$ & $0.306^{* *}$ & $0.151^{*}$ & -0.067 & -0.068 & -0.033 & $0.415^{* *}$ & $0.407 * *$ & 1 & & & \\
\hline NDHL & $0.283^{* *}$ & $0.168^{*}$ & $0.305^{* *}$ & $0.142^{*}$ & -0.076 & -0.078 & -0.041 & $0.416^{* *}$ & $0.408^{* *}$ & $0.998 * *$ & 1 & & \\
\hline DPL & $0.261^{* *}$ & 0.022 & $0.205^{* *}$ & 0.043 & -0.115 & -0.090 & 0.010 & $0.178^{*}$ & $0.181^{*}$ & $0.437 * *$ & $0.447 * *$ & 1 & \\
\hline NDPL & $0.269^{* *}$ & 0.022 & $0.200^{* *}$ & 0.042 & -0.114 & -0.090 & 0.012 & $0.170^{*}$ & $0.174 *$ & $0.435^{* *}$ & $0.446 * *$ & $0.996^{* *}$ & 1 \\
\hline
\end{tabular}

BH - body height, BW - body weight, BMI - body mass index, DHGS - dominant handgrip strength, NDHGS - non dominant handgrip strength, DHS - dominant hand span, NDHS - non dominant hand span, DHL - dominant hand length, NDHL - non dominant hand length, DPL - dominant palm length and NDPL - non dominant palm length.

between DHGS and NDHGS. Therefore, $\mathrm{H}_{0}$ was rejected, implying that handedness has the ability to generate higher HGS among Nigerian school children.

\section{Hypothesis 3}

There will be no significant relationships between hand length and HGS, or palm length and HGS, among Nigerian school children.
A disproportionate and insignificant correlation ( $p>0.05$ ) was found between dominant hand length (DHGS, r $=-0.068$; NDHGS, $r=-0.033$ ) and HGS as well as non-dominant hand length (DHGS, $\mathrm{r}=-0.078$; NDHGS, $r=-0.041)$ and HGS. The $\mathrm{H}_{0}$ was therefore retained. This implies that longer hand length does not affect HGS among Nigerian primary school students. It was also 
uniquely observed from this study that a disproportionate (DPL, $r=-0.090$; NDPL, $r=-0.090$ ) and insignificant $(p>0.05)$ relationship exists between dominant and nondominant palm length and DHGS. Also, a proportionate (DPL, $r=0.010$; NDPL, $r=0.012$ ) but insignificant $(p>0.05)$ relationship exists between dominant and nondominant palm length and NDHGS. Therefore, $\mathrm{H}_{0}$ was retained, implying that hand length cannot determine HGS among Nigerian school children and any relationship noticed can be attributed to the chance factor.

\section{Hypothesis 4}

There will be no significant association between hand span and HGS among Nigerian school children.

This study showed that DHS and NDHS are disproportionate (DHS, $r=-0.177$; NDHS, $r=-0.182$ ) and significant $(\mathrm{p}<0.05)$ correlates of DHGS. Also, a disproportionate (DHS, $r=-0.100$; NDHS, $r=-0.103$ ) but insignificant $(p>0.05)$ relationship exists between DHS and NDHS, and NDHGS. Therefore, $\mathrm{H}_{0}$ was rejected, implying that hand span can determine DHGS only among Nigerian school children.

\section{Hypothesis 5}

Gender will not significantly influence HGS among Nigerian school children.

It was observed from this study that a significant difference $(p>0.05)$ exists between DHGS and NDHGS across gender characteristics. Therefore, $\mathrm{H}_{0}$ was rejected, implying that a gender difference does influence HGS among Nigeria school children.

\section{Discussion}

The main outcome of this study indicated that of all the anthropometric parameters analyzed, only BMI, handedness and hand span correlated significantly with HGS. This study showed that BMI is a significant correlate of HGS. This suggests that BMI influences HGS. Related studies also showed that low BMI is associated with poor HGS and vice versa $[3,16]$. The implication of this is that being younger may be responsible for recording low BMI because of the low percentage of muscle mass resulting in poor HGS. Handedness was found to relate positively and significantly to HGS in this study. This is because the constant use of a particular hand tends to be stronger according to the principle of reversibility (use it or lose it). This finding is supported by a study reporting that HGS is affected by handedness [2] and also by another similar study which concluded that dominant hand is significantly and proportionately related to HGS [17].

Furthermore, hand length was observed to associate disproportionately and insignificantly with HGS in this study. This finding is not in agreement with the previous studies $[14,15,17]$. This disagreement might not be unconnected to variation in study methodology including subject characteristics and differences in measuring instruments of HGS, among others. Therefore, according to this study longer hand length is not a predictor of better HGS. Also, palm length was found to correlate disproportionately with DHGS but proportionately with NDHGS, although all are insignificant. In contrast, a previous study showed that persons with longer fingers and larger hand surfaces or longer palm length have stronger grip power in all age groups [4]. These contrasting findings might also be due to variation in study methodology.

A disproportionate relationship was found between hand span and HGS, although it was only significant with DHGS. This finding is not in total agreement with a past study [1]. The implication of this outcome is that a wider hand span can only influence DHGS among Nigerian primary school students. This is because the constant use of a particular hand tends to make it stronger or wider according to the principle of reversibility (use it or lose it). Also, the significant difference between gender characteristics and HGS suggests that gender is associated with HGS and vice versa among Nigerian school children, as also reported in other studies elsewhere $[8,9,11]$. The variation in percentage of skeletal muscle mass development between boys and girls could be responsible for total body strength and by implication affect the execution of some skills requiring the use of the hand and involving physical demands. However, this could simply reflect the sexual dimorphism, due to the action of sex steroid hormones.

However, the major limitation of this study was that the parental socioeconomic status of the participants, which could determine some anthropometric measures, was not taken into consideration.

\section{Conclusions}

This study therefore concluded that the most important determinants of HGS among children are BMI, handedness and hand span, and thus could be considered as markers of nutritional and health status, as well as physical fitness of these individuals.

It is expected that the findings of this study:

- may be useful in the process of talent identification in sports involving the use of handgrip;

- might serve as an index for the government and healthcare providers in policy making as regards the relationship between anthropometrics and HGS and also as a marker of nutritional and health status as well as physical fitness;

- may also establish a simple model to predict maximal grip strength in the dominant hand. 
It is therefore recommended that longitudinal studies should be conducted to ascertain the relative effects of growth on HGS, and also future larger studies are needed to confirm the findings of this study.

Conflict of interest: Authors state no conflict of interest.

\section{References}

1. Bansode D.G., L.J. Borse, R. Yadav (2014) Study of correlation between dominant hand's grip strength and some physical factors in adult males and females. Int. J.. Pharm. Res. Health Sci., 2(4): 316-323.

2. Baskaran C., G. Arindam, P. Chandan, C. Bidhan (2010) Anthropometric traits predict hand grip strength in healthy normal. Springer J. Hand .Microsurg., 2(2): 58-61.

3. Das A., M. Dutta (2015) Correlation between body mass index and handgrip strength and handgrip endurance among young healthy adults. J. Evid. Based Med. Healthcare, 2: 3995-4001.

4. Fallahi A.A., A.A. Jadidian (2011) The Effect of Hand Dimensions, Hand Shape and Some Anthropometric Characteristics on Handgrip Strength in Male Grip Athletes and Non-Athletes. J. Hum. Kinet., 29: 151-159.

5. Fess E.E. (1992) Grip Strength. American Society of Hand Therapists. 2nd edition, Chicago.

6. Flood A., A. Chung, H. Parker, V. Kearns, T.A. O'Sullivan (2014) The use of hand grip strength as a predictor of nutrition status in hospital patients. Clin. Nutr., 33: 106-114.

7. Guerra R.S., I. Fonseca, F. Pichel, M.T. Restivo, T.F. Amaral (2013) Handgrip strength and associated factors in hospitalized patients. J. Parenter. Enteral. Nutri., Published Online November 29, 0148607113514113.

8. Hogrel J.Y., V. Decostre, C. Alberti, A. Canal, G. Ollivier, E. Josserand (2012). Stature is an essential predictor of muscle strength in children. BMC Musculoskelet Disord., 13: 176
9. Incel N.A., E. Ceceli, P.B. Durukan, H.R. Erdem, Z.R. Yorgancioglu (2002) Grip strength: Effect of hand dominance. Singapore Med. J., 43(5): 234-237.

10. Jürimäe T., T. Hurbo, J. Jurimae (2009) Relationship of handgrip strength with anthropometric and body composition variables in prepubertal children. Homo., 60: 225-238.

11. Koley S., A.P. Singh (2009) An association of dominant hand grip strength with some anthropometric variables in Indian collegiate population. Anthropologischer Anzeiger, 67: 21-28.

12. Milenkovic S., M. Dragovic (2013) Modification of the Edinburgh Handedness Inventory: a replication study. Laterality, 18(3): 340-348.

13. Norman K., N. Stobäus, M.C. Gonzalez, J.D. Schulzke, M. Pirlich (2011) Hand grip strength: outcome predictor and marker of nutritional status. Clin. Nutr., 30: 135-142.

14. Ross C.H., B. Rösblad (2002) Norms for grip strength in children aged 4-16 years. Acta Paediatr., 91(6): 617-625.

15. Shyamal K., P.K. Satinder (2011).Colligiate of hand grip strength in selected hand-arm-anthropometric variables in Indian inter-university female volleyball players. Asian J. Sports Med., 2(4): 220-226.

16. Vaz M., S. Hunsberger, B. Diffey (2002) Prediction equations for handgrip strength in healthy male and female subjects encompassing a wide age range. Ann. Hum. Biol., 29: 131-141.

17. Visnapuu M., T. Jurimae (2007) Handgrip strength and hand dimensions in young handball and basketball players. J. Strength Cond. Res., 21(3): 923-929.

\section{Received 11.01.2017 \\ Accepted 10.04.2017}

(c) University of Physical Education, Warsaw, Poland 